\title{
PREFACE TO THE SPECIAL ISSUE ON RECENT PROGRESS IN 3-D VISUAL PERCEPTION OF ROBOTS
}

\author{
Piotr SKRZYPCZYŃSKI *, Tomasz KORNUTA †
}

This special issue of the Foundations of Computing and Decision Sciences, titled "Recent progress in 3-D visual perception of robots", is devoted to three- dimensional (3-D) perception with active range sensors applied in various robotic tasks. The introduction of Microsoft Kinect RGB-D sensor few years ago triggered a great progress in the 3 -D perception research in robotics. Nowadays, the researchers can choose from several types of commodity RGB-D sensors, which are compact, lightweight, and affordable. Fast and reliable depth measurements of the analyzed scene provided by the RGB-D cameras allow to solve many of the problems inherent to the solutions based on traditional passive vision. Although the off-the-shelf RGB-D cameras are mainly limited to indoor use, the recently introduced compact 3-D laser scanners, such as Velodyne VLP-16, enable progress in 3-D robotic perception outdoors.

The special issue contains six papers being extended versions of papers presented at the special session "Perception of robots based on RGB-D images 2" organized during the 14th National Conference on Robotics in Polanica Zdrój, Poland. The conference was held from 14th till 18th of September 2016, and it was organized by the Department of Fundamental Cybernetics and Robotics, Electronics Faculty, Wroclaw University of Technology. This special session was conceived by Tomasz Kornuta as a follow-up event after the successful special session under the same title that took place at the National Conference on Robotics in 2014. The main idea behind these events was to provide a forum where Polish researchers, working in various computer science sub-fields including computer vision and robotics, could present and discuss recent achievements, challenges, and new application areas of 3-D perception for robots. Thirteen papers were presented at the 2016 special session, covering a wide range of robot applications, sensing techniques, and data processing concepts.

The idea of publishing a selection of these papers in a journal emerged at the planning stage of the session. Robotics research is a particularly interdisciplinary field of science and engineering. Researchers in robotics build complicated systems

\footnotetext{
*Poznań University of Technology, Institute of Control, Robotics and Information Engineering, Piotrowo 3A, 60-965 Poznań, piotr.skrzypczynski@put.poznan.pl

†IBM Research, Almaden, 650 Harry Rd, San Jose, CA 95120, tkornut@us.ibm.com
} 
that often embody machine intelligence and software engineering paradigms into sophisticated hardware, drawing ideas, methodology, and particular techniques from computer science, control theory, mechanics, machine learning, soft computing and many other areas of the engineering science. Due to the unavoidable uncertainty of physical measurements, the practical problems encountered in 3-D robotic perception must often involve approximate reasoning and lead to novel, interesting approaches to diverse problems, including object recognition, scene understanding, etc. Therefore, we have looked for an established international journal that could serve as a vehicle ensuring world-wide dissemination of the results and ideas presented at the special session. The journal of Foundations of Computing and Decision Sciences (FCDS), that until 1990 was known as Foundations of Control Engineering, and focuses on the links between Computing and broadly understood Decision Sciences, thus seemed to be a very interesting choice. We hope that publishing this collection of papers in FCDS not only ensures the high scientific and editorial quality for our project, but also contributes to a wider recognition of the achievements of Polish roboticists in the computer science community.

The papers contained in this special issue are improved, and often much extended versions of the conference papers. The results have been described more comprehensively, in a wider context of the related work and often included new experiments, taking into account the outcome of the extensive discussions at the special session. Authors of the selected papers, recommended by the Program Committee of the National Conference on Robotics, were invited to submit their works to the journal. All contributions were subjected to the regular FCDS review procedure. Finally, six papers were accepted for the special issue.

At that point we would like to express our gratitude to all of the reviewers who provided insightful comments, enabling significant improvement of the manuscripts and made the accepted papers more clear and accessible to the readers.

The six papers presented here focus on key issues in 3-D perception for both manipulators and mobile robots, namely: acquisition and registration of RGB-D data that describe the objects of manipulation, recognition and classification of objects described by 3-D data, scene classification, robot localization, and efficient parallel processing of $3-\mathrm{D}$ data.

The work entitled "ModReg: A Modular Framework for RGB-D Image Acquisition and 3D Object Model and Registration" by Kornuta and Stefańczyk, which opens the special issue, addresses the problem of registration of 3-D models of objects for applications requiring object recognition, such as intelligent manipulation. A multicamera setup supplemented with a pattern projector is used to acquire high-resolution RGB-D images, which are then post-processed into dense point cloud models. An important innovation is the use of a graph-based optimization technique derived from the state-of-the-art mobile robot navigation algorithms to obtain consistent 3-D object models from separate views provided by the cameras.

The next paper by Koguciuk, entitled "Parallel RANSAC for Point Cloud Registration", also tackles the problem of registration of massive RGB-D data into consistent point clouds. This work focuses on a particular component of the point cloud registration system - the Random Sampling Consensus (RANSAC) procedure, which is 
typically used to eliminate outliers while registering data to a mathematical model. As RANSAC is based on the idea of sampling at random the possible solutions, it easily becomes a bottleneck in the registration system if the registered data are massive. Therefore, Koguciuk introduces a parallelized RANSAC algorithm and demonstrates its efficient implementation in the CUDA architecture. The experimental results show that exploring this parallel computing solution it is possible to find local coordinate systems of objects in the scene nearly in real-time.

With the work of Czajewski and Kołomyjec, entitled "3D Object Detection and Recognition for Robotic Grasping Based on RGB-D Images and Global Features", we stay within the application area related to robotic manipulation, but the considered problems are shifted from the fundamental issues of registration of the RGB-D data to the level of 3-D object detection and recognition. Objects observed by a RGB-D sensor are identified based on both geometric and visual cues, and then their color histograms are considered. This approach enables distinguishing objects of similar geometry but different visual appearance. The recognized objects are then localized in the 3-D space and autonomously grasped by a manipulator. The experiments, conducted in a domestic-like environment, proved the capability of the robotic system to grasp everyday items like soda cans, coffee mugs, etc.

The paper "Keypoint-less, Heuristic Application of Local 3D Descriptors" written by Harasymowicz-Boggio and Chechliński takes the reader away from object-level representations characteristic to grasp-related applications. Instead, this paper proposes a keypoint-less approach to attention focusing, where the full scene is processed in a hierarchical manner. The whole scene point clouds are processed by weaker but faster classifiers, and their results are then used as heuristics for extraction of increasingly robust descriptors. This novel approach was validated on realistic indoor scenes using a massively- parallel, open source object recognition system developed by the authors, and it has a potential to be used in both manipulation and mobile robotics applications.

Mobile robotics is the target application area of the next work, titled "Place Classification Using Dempster-Shafer Theory" by Siemiątkowska and Harasymowicz-Boggio. The paper presents a novel place classification method, where places visited by a robot equipped with a RGB-D sensor are labelled according to the classes of objects recognized at these locations. The recognized objects vote for a set of possible place classes. The votes are aggregated using the Dempster-Shafer theory, which allows this method to take into account the uncertainty of data. An important advantage of the proposed algorithm is the ability to relax the closed world assumption, as the classification system can give the "unknown" class label as an answer, whenever the supporting evidence is weak.

The final paper by Będkowski, Röhling, Hoeller, Schulz and Schneider, entitled "6D Simultaneous Localisation and Mapping Algorithms for Robotic Mobile Mapping Systems", also deals with mobile robotics, but it transports the reader from clean lab setups into a real outdoor environment. The paper is a study of 6D SLAM algorithms - here 6D refers to the six degrees of freedom of the moving sensor. Various data registration and loop closing methods are evaluated, focusing on two new aspects: the environment semantics-based discrimination of the measured points, and the parallel 
computing techniques based on CUDA architecture used to improve the processing speed of the selected components. Moreover, a novel method, based on ground truth data obtained by geodetic survey, is used for quantitative evaluation of the 6D SLAM system.

As witnessed by these papers, the Polish robotics community is deeply interested in research directions that lie on the crossroads between the "classic" robotics, strongly rooted in mechanics and control theory, and the approach, which fully explores the syncretic nature of modern robotics, being able to pick the necessary building blocks from various sources, still retaining the rigorous methodological framework of an engineering science. This selection of six papers shows also the potential diversity of those research directions. All the papers presented here were authored or coauthored by young researchers, who simply enjoy creating things that are robust and really work in the real world. Both the variety of problems considered here, and the natural need of the young researchers to exchange ideas and gain experience motivate us to continue the efforts aimed at providing the community with next opportunities for focused discussions on 3-D perception, mapping, modelling, reasoning, and other aspects of robotics, that will for sure soon become " $3-\mathrm{D}$ ".

Last but not least, we would like to express our deepest gratitude to the people, who made possible bringing our ideas to existence: Prof. Krzysztof Tchon, the chair of the National Conference on Robotics, who provided an excellent and friendly environment for the special session, and Prof. Jerzy Stefanowski, the editor-in-chief of FCDS, who decided to let in robotics into the computing-oriented journal, as well as supported us with his experience through the whole process of preparing the special issue.

Tomasz Kornuta wants also to thank his managers and peers in IBM Research, Almaden, namely Dr. Winfried Wilcke and Dr. Ahmet S. Ozcan, former and present managers of the Machine Intelligence Team, and Dr. Chandrasekhar Narayan, Director for Science and Technology, who not only kindly agreed to financially support his participation in the National Conference on Robotics, but also enabled him to pursue his interests in 3-D robot perception and involvement in the organization of the event.

Obviously, we also would like to thank all the authors. This project came to life only because of your hard work.

Guest Editors:

Piotr Skrzypczyński (Poznań University of Technology)

Tomasz Kornuta (IBM Research, Almaden) 